\title{
Will new drugs cure acute myeloid leukaemia?
}

\author{
Robert Peter Gale MD \\ Centre for Haematology, Department of Immunology and Inflammation, Imperial College London, London, UK \\ Robert Peter Gale MD, PhD, DSc (hc), FACP, FRCPI (hon), \\ FRSM, Centre for Haematology Research, Department of \\ Phone: +19086560484 \\ Immunology and Inflammation, Imperial College London, \\ Fax: +13103881320 \\ E-Mail: robertpetergale@alumni.ucla.edu
}

London, UK SW7 2AZ

Citation: Gale RP. Will new drugs cure acute myeloid leukaemia? Cell Ther Transplant 2021; 10(3-4): 4-7.

\section{Summary}

There are many new therapies approved to treat acute myeloid leukaemia (AML) including conventional and targeted drugs, and immune therapy. Most improve diverse outcomes including event- and relapse-free survivals and survival. However, most effect sizes are small and failure rates by 2 years are high. Based on the data reviewed above I conclude: (1) many new AML therapies target specific AML sub-types; (2) none are proved better than intensive radiochemotherapy in persons who could receive either therapy; (3) there is disagreement defining who can or cannot receive intensive therapy;
(4) there are important problems with several new drug approvals; (5) azacitidine and venetoclax may be the new standard-of-care in elderly persons with AML judged unable to receive intensive therapy; and (6) new drugs are welcome but have not had a big impact on long-term survival of most people with AML.

\section{Keywords}

Acute myeloid leukemia, targeted therapy, efficiency.

\section{Introduction}

There are many new therapies approved to treat acute myeloid leukaemia (AML) including new conventional and targeted drugs and immune therapy. A summary of new AML drugs is displayed in Figure 1 increasing from one approval every 6 years to one approval every 150 days, a 12-fold increase.

The question I consider is whether these new therapies will cure AML. My discussion is divided into 3 categories: (1) conventional drugs; (2) targeted therapies; and (3) immune therapy.

\section{New drugs}

I consider 4 new drugs: (a) venetoclax [1]; (b) CPX-351 [2]; (c) CC-486 [3]; and (d) glasdegib ( \pm low-dose cytarabine) [1-4]. Results of these, quite recent trials demonstrate that, although each new drug, alone or combined with previouslyapproved drugs improved outcomes, there remains a high rate of failures by 2 years.

\section{Targeted drugs}

Four targeted drugs are approved in AML including: (1) midostaurin; (2) gilteritinib; (3) enasidenib, and (4) ivosidenib. Results of recent trials of these 3 drugs are published, and the survival curves can be compared [5-8]. Except for enasidenib, these drugs improve outcomes but 2-year failure rates are high. A US trial Beat AML in persons with newly-diagnosed AML assigned subjects with druggable mutations to targeted or conventional drugs. There was no important difference in outcomes [9]. Therefore, according to recent estimates, current targeted drugs are likely to help only a limited subgroup (ca. 10 percent) of patients with acute myeloid leukemia [10].

\section{Immune therapy}

Gemtuzumab, an anti-CD33 monoclonal toxin-linked antibody, is the only approved immune therapy of AML [11]. It modestly improves outcomes and is rarely used. 


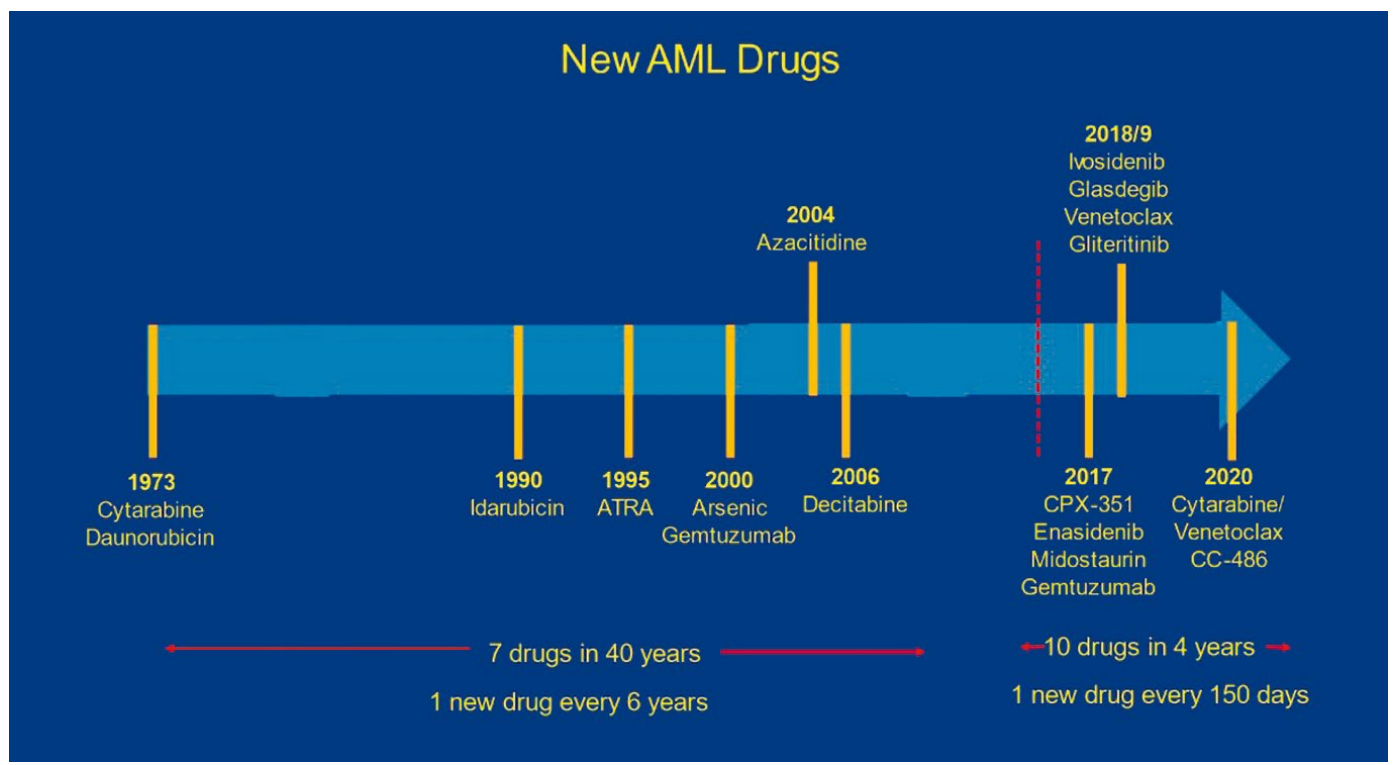

Figure 1. Drug approvals in AML 1973-2020

\section{Issues in new drug approvals}

Several important issues confound analyses of the appropriate use of new drugs in AML including: (1) who is unfit for intensive therapy? (2) no randomized trial proves less-intensive therapy is better than conventional intensive therapy amongst persons who could receive either; (3) what is the best endpoint for new drug approvals; (4) what is the appropriate comparator for a new drug approval; (5) several recent approvals are for unstudied populations; (6) recent approvals will decrease enrollment in clinical trials; and (7) most new drugs improve survival only slightly and long-term results remain unsatisfactory [12]. Table 1 displays data indicating not everyone benefits from a new drug such as venetoclax [1].

Table 1. Differential effects of azacitidine and venetoclax in sub-cohorts

\begin{tabular}{|l|l|l|}
\hline \multicolumn{3}{|c|}{ Who benefits from azacitidine + venetoclax } \\
\hline & Benefit & No benefit \\
\hline Sex & Female & Male \\
\hline Age & $\geq 75 \mathrm{y}$ & $<75 \mathrm{y}$ \\
\hline Country & US/EU & Russia/China/Japan \\
\hline ECOG PS & 1 & $\geq 2$ \\
\hline Cytogenetics & Intermediate & Poor \\
\hline Mutations & IDHI/IDH2 & FLT3/TP53/NPM1 \\
\hline MDS changes & No & Yes \\
\hline $\begin{array}{l}\text { Bone marrow } \\
\text { blasts }\end{array}$ & $\geq 30 \%$ & $<30 \%$ \\
\hline
\end{tabular}

Table 2 shows although azacitidine and venetoclax improve survival of older persons with AML there remains a major loss of potential life-expectancy. Finally, Table 3 displays the cost of several new AML drugs compared with conventional drugs.
Table 2. Impact of azacitidine and venetoclax on reversing loss in life-expectancy

\begin{tabular}{|l|l|l|l|l|}
\hline \multicolumn{5}{|c|}{ Remaining life-expecancy } \\
\hline Age & $\begin{array}{l}\text { Expected } \\
\text { age }\end{array}$ & $\begin{array}{l}\text { Added } \\
\text { years }\end{array}$ & $\begin{array}{l}\text { Venetoclax } \\
\text { azacitidine }\end{array}$ & $\begin{array}{l}\text { Lost } \\
\text { years }\end{array}$ \\
\hline Male 75 & 86 & 11 & 2 years & 9 \\
\hline Female 75 & 89 & 14 & 2 years & 12 \\
\hline
\end{tabular}

Table 3. Relative per year costs of some new drugs

\begin{tabular}{|l|l|}
\hline \multicolumn{2}{|c|}{ Cost per Cycle or 1-Year (USD) } \\
\hline Cytarabine/Daunorubicin & $\$ 1,500$ \\
\hline Azacitidine & $\$ 4,400$ \\
\hline Cilteritinib & $\$ 105,000$ \\
\hline Enasidenib & $\$ 120,000$ \\
\hline
\end{tabular}

\section{Conclusions}

Based on the data I review above I conclude: (1) many new AML therapies target specific AML sub-types; (2) none are proved better than intensive radiochemotherapy in persons who could receive either therapy; (3) there is disagreement defining who can or cannot receive intensive therapy; (4) there are important problems with several new drug approvals; (5) azacitidine and venetoclax may be the new standard-of-care in elderly persons with AML judged unable to receive intensive therapy; and (6) new drugs are welcome but have not had a big impact on long-term survival of most people with AML.

\section{Acknowledgement}

Presented in part at the Raisa Gorbacheva Symposium in St. Petersburg on 17 September 2021. RPG acknowledges support from the National Institute of Health Research (NIHR) Biomedical Research Centre funding scheme. 


\section{Conflict of interest}

RPG is a consultant to: BeiGene Ltd., Fusion Pharma LLC, LaJolla NanoMedical Inc., Mingsight Pharmaceuticals Inc. and CStone Pharmaceuticals. Medical Director of FFF Enterprises Inc, on the Board of Directors: RakFond Foundation for Cancer Research Support. Scientific Advisory Board: Antegene Biotech LLC, StemRad Ltd. Author Contribution: I conceived, wrote and submitted the typescript for publication. Ethics Approval: None required.

\section{References}

1. DiNardo CD, Jonas BA, Pullarkat V, Thirman MJ, Garcia JS, Wei AH, et al. Azacitidine and Venetoclax in previously untreated acute myeloid leukemia. N Engl J Med. 2020;383(7):617-629. doi: 10.1056/NEJMoa2012971

2. Lancet JE, Uy GL, Newell LF, Lin TL, Ritchie EK, Stuart RK, et al. CPX-351 versus 7+3 cytarabine and daunorubicin chemotherapy in older adults with newly diagnosed highrisk or secondary acute myeloid leukaemia: 5-year results of a randomised, open-label, multicentre, phase 3 trial. Lancet Haematol. 2021;8(7):e481-e491. doi: 10.1016/S23523026(21)00134-4. PMID: 34171279

3. Wei AH, Döhner H, Pocock C, Montesinos P, Afanasyev B, Dombret H, et al.; QUAZAR AML-001 Trial Investigators. Oral Azacitidine maintenance therapy for acute myeloid leukemia in first remission. N Engl J Med. 2020; 383(26):25262537. doi: 10.1056/NEJMoa2004444

4. Cortes JE, Heidel FH, Hellmann A, Fiedler W, Smith $\mathrm{BD}$, Robak T, et al. Randomized comparison of low dose cytarabine with or without glasdegib in patients with newly diagnosed acute myeloid leukemia or high-risk myelodysplastic syndrome. Leukemia. 2019; 33(2):379-389. doi: 10.1038/s41375-018-0312-9

5. Stone RM, Mandrekar SJ, Sanford BL, Laumann K, Geyer S, Bloomfield CD, et al. Midostaurin plus chemotherapy for acute myeloid leukemia with a FLT3 mutation. N Engl J Med. 2017; 377(5):454-464. doi: 10.1056/NEJMoa1614359

6. Perl AE, Martinelli G, Cortes JE, Neubauer A, Berman E, Paolini S, et al. Gilteritinib or chemotherapy for relapsed or refractory FLT3-mutated AML. N Engl J Med. 2019; 381(18):1728-1740. doi: 10.1056/NEJMoa1902688

7. DiNardo CD, Schuh AC, Stein EM, Montesinos P, Wei $\mathrm{AH}$, de Botton S. Enasidenib plus azacitidine versus azacitidine alone in patients with newly diagnosed, mutant-IDH2 acute myeloid leukaemia (AG221-AML-005): a single-arm, phase $1 \mathrm{~b}$ and randomised, phase 2 trial. Lancet Oncol. 2021; 22(11):1597-1608. doi: 10.1016/S1470-2045(21)00494-0

8. Roboz GJ, DiNardo CD, Stein EM, de Botton S, Mims AS, Prince GT, et al.. Ivosidenib induces deep durable remissions in patients with newly diagnosed IDH1-mutant acute myeloid leukemia. Blood. 2020 Feb 13;135(7):463-471. doi: 10.1182/blood.2019002140

9. Burd A, Levine RL, Ruppert AS, Mims AS, Borate U, Stein EM, et al. Precision medicine treatment in acute myeloid leu- kemia using prospective genomic profiling: feasibility and preliminary efficacy of the Beat AML Master Trial. Nat Med. 2020; 26(12):1852-1858. doi: 10.1038/s41591-020-1089-8

10. Prasad V, Gale RP. Precision medicine in acute myeloid leukemia: Hope, hype or both? Leuk Res. 2016; 48:73-77. doi: 10.1016/j.leukres.2016.07.011

11. Lambert J, Pautas C, Terré C, Raffoux E, Turlure P, Caillot $\mathrm{D}$, et al. Gemtuzumab ozogamicin for de novo acute myeloid leukemia: final efficacy and safety updates from the open-label, phase III ALFA-0701 trial. Haematologica. 2019; 104(1):113-119. doi: 10.3324/haematol.2018.188888

12. Estey E, Karp JE, Emadi A, Othus M, Gale RP. Recent drug approvals for newly diagnosed acute myeloid leukemia: gifts or a Trojan horse? Leukemia. 2020;34(3):671-681. doi: 10.1038/s41375-019-0704-5 


\section{| Будут ли новые лекарства излечивать острый миелоидный лейкоз?}

\section{Роберт П. Гэйл}

Центр гематологии, Департамент иммунологии и воспаления, Лондонский Имперский Колледж, Лондон, Великобритания

\section{Резюме}

Существует много новых методов лечения, одобренных для лечения острого миелоидного лейкоза (ОМЛ), включая традиционные и таргетные препараты, а также иммунотерапию. Большинство из них улучшают различные исходы, включая бессобытийную и безрецидивную выживаемость. Однако в большинстве случаев выраженность эффекта невелика, и высока частота неуспешной терапии при 2-летнем наблюдении. Основываясь на данных, рассмотренных выше, сделаны выводы о том, что: (1) многие новые методы лечения ОМЛ направлены на терапию определенных подтипов ОМЛ; (2) ни один из них не оказался лучше, чем интенсивная химиолучевая терапия пациентов, которые могли бы получать любой из этих видов лечения;
(3) существуют разногласия по поводу того, кто может или не может получать интенсивную терапию; (4) существуют серьезные проблемы с одобрением нескольких новых лекарственных препаратов; (5) азацитидин и венетоклакс могут быть новым стандартом лечения пожилых людей с ОМЛ, признанных неспособными получать интенсивную терапию; и (6) новые препараты должны рассматриваться, но пока не оказали большого влияния на долгосрочное выживание большинства пациентов с ОМЛ.

\section{Ключевые слова}

Острый миелоидный лейкоз, таргетная терапия, эффективность. 